\title{
Analyzing the Credit Default Swap Market Using Cartesian Genetic Programming
}

\author{
Laleh Zangeneh and Peter J. Bentley \\ Department of Computer Science \\ University College London, London WC1E 6BT, UK. \\ Email: \{L.Zangeneh, P.Bentley\}@cs.ucl.ac.uk
}

\begin{abstract}
The credit default swap has become well-known as one of the causes of the 2007-2010 credit crisis but more research is vitally needed to analyze and define its impact more precisely and help the financial market transparency. This paper uses cartesian genetic programming as a discovery tool for finding the relationship between credit default swap spreads and debts and studying the arbitrage channel. (Arbitrage is the practice of taking advantage of a price difference between markets.) To our knowledge this work is the first attempt toward studying the credit default swap market via an evolutionary process and our results prove that cartesian genetic programming is human competitive and it has the potential to become a regression discovery tool in credit default swap market.
\end{abstract}

Key words: Cartesian Genetic Programming, Credit Default Swap, Regression.

\section{Introduction}

In the last two years, the world economy has been faced with one of the biggest crises ever seen, throwing most countries into recession. The causes of the financial meltdown are numerous, but it is widely accepted that one significant factor was the "Credit Default Swap" market. Trading of this complex financial product was unpredictable, out of control, and badly priced, leading to fortunes being made and lost [11].

To understand what a Credit Default Swap (CDS) really is, consider the following example. Imagine North bank made a five-year $\$ 10$ million loan to West Airways. North bank is concerned about West Airways performance and not being able to pay back the loan (possible default). Therefore, in order to protect itself and reduce the risk of not getting its loaned money back, North bank can buy a kind of insurance (known as "protection") on West Airways from a insurance seller (a protection seller), which in this case might be East bank. The insurance is based on a West Airways-issued bond (a debt security which represents a formal contract to repay borrowed money with interest at fixed intervals). This protection (insurance) contract is called a CDS contract and East bank is then able to trade its CDS contracts with other banks, buying 
them when they cost less and selling them when they are worth more, in order to make profit. The price of CDS contract changes according to the success or failure of the business of West Airways (i.e., the credit quality of West Airways). If the West Airways credit quality decreases (risk of default increases), the CDS price will increase.

One of the major reasons why this product help cause the financial crisis is because CDS contracts were often very poorly costed-although they were supposed to represent a kind of insurance against a loan, their prices often showed little relationships to the true ability of the companies to repay those loans. Thus when companies unexpectedly defaulted on their loans (or unexpectedly paid the loans back), a bank that bought a CDS contract at a very high price might suddenly find it was worth very little, and it would lose money. Thus the pricing of CDS contracts is of enormous concern and consequence. In this paper, the first ever study is performed into CDS pricing by using Cartesian Genetic Programming to analyse the relationship between price, debt and equity information. We show that CGP can completely outperform the standard pricing model, and we provide some analysis of the CGP solution, as well as the ability of CGP to cope with this complex financial data.

More details of CDS contract pricing is provided in the following section. This is followed by a description of our CGP model in section three. Section four explains the datasets, CGP settings and experiment objectives. Results are provided in the fifth section and we conclude in section six.

\section{Credit Default Swap Background}

A CDS contract is a kind of credit derivative. Credit derivatives are over-thecounter (OTC $)^{1}$ financial contracts that allows one to take or reduce credit exposure, commonly on bonds or loans of corporate entity and it reflects the risk of a default in a corporation. This risk is expressed through the CDS price. A CDS is an agreement between two parties to exchange the credit risk of a reference entity, also called an issuer (West Airways, in our previous example), without directly involving the issuer [1]. The protection buyer (North bank, in our example) pays a periodic fee and receives compensation if the reference entity has a credit event. A credit event includes bankruptcy, failing to pay outstanding debt obligations, or restructuring of a bond or loan. The protection seller (East bank) collects the periodic fee and profits if the credit of the reference entity remains stable or improves while the swap is outstanding [2]. Figure 1 illustrates the terminology and mechanism of the CDS. The CDS is uniquely defined by four key parameters [1-3]:

1. Issuer: CDS contracts specify a reference bond or loan which defines the issuing entity through the bond prospectus (e.g. West Airways).

\footnotetext{
1 The phrase "over-the-counter" can be used to refer to stocks, debt securities and other financial instruments such as derivatives, which are traded through a dealer as opposed to on a centralized exchange (e.g. London Stock Exchange).
} 


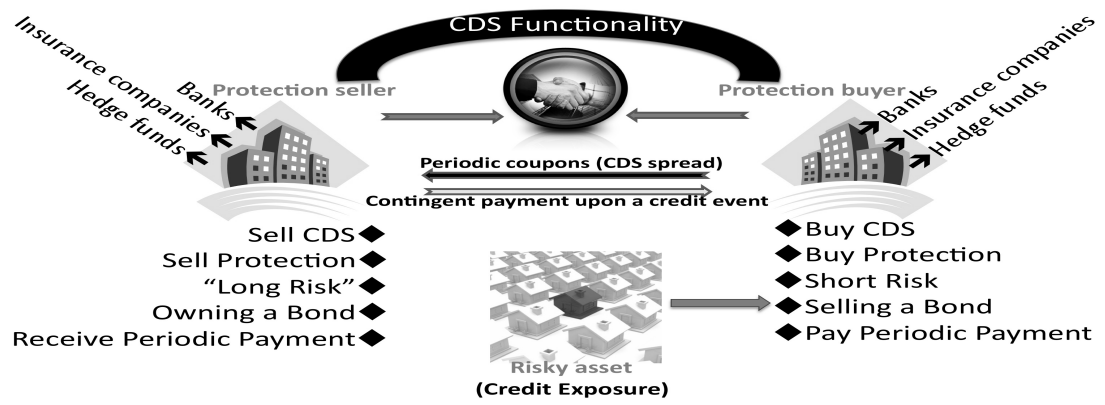

Fig. 1: Single Name CDS Functionality

2. Notional amount: Notional amount is the amount of credit risk being transferred between protection buyer (North bank) and protection seller (East bank).

3. Spread: A spread (also called coupon, or price) specifies the annual payments which are quoted in basis point ${ }^{2}$. These payments are paid quarterly (e.g. from North bank to East bank).

4. Maturity Date: The expiration of the contract. The most liquid ${ }^{3}$ maturity term for CDS contract is 5 years.

Over the last few years, the credit derivatives market has grown significantly and exceeds both equity derivatives and corporate bond markets. The largest participants in the credit derivatives market are banks, insurance and securities companies. According to the British Bankers Association (BBA) report, the most important and widely used products in credit derivatives are CDS $(42 \%$ of notional principal outstanding in 2006) [2]. The reality shows that the CDS market suffers from a lack of any comprehensive study. The lack of sufficient data had been a major problem for a broad empirical testing of CDS pricing models (as seen in $[7,8]$ ) until few years ago.The last few years with increased bond market liquidity and a well-developed CDS market provide more sufficient data for investigation.

\subsection{CDS Pricing Challenge}

In the credit risk literature, there are two broad approaches to modelling corporate default risk (e.g. the risk that West Airways defaults on a loan): the structural approach and the reduce-form model. In the structural model the evolution of the company's assets follows the diffusion process. In other words the default occurs when the value of the firm assets becomes lower than its debts; because the assets can be continuously assessed, downwards trends can be spotted and so the risk of default should never be a surprise. In contrast to the structural approach, the reduce-form approach assumes that there is no relation between

\footnotetext{
${ }^{2}$ A basis point (often denoted as $\mathrm{bp}$ ) is a unit relating to interest rates that is equal to $1 / 100$ th of a percentage point per annum (pa).

${ }^{3}$ Liquid means easily converted into cash (e.g. a bond which can be sold quickly).
} 
value of the company and risk of default. In this approach defaults are seen as an unpredicted Poisson events involving a sudden loss in market value and therefore firms never default gradually. See $[4-6,11]$ for more details on credit risk literature. Table 1 presents the history of credit risk approaches by referring to the investigators who contributed to the field.

\begin{tabular}{|c|c|c|c|}
\hline \multicolumn{2}{|l|}{ Structural Model } & \multicolumn{2}{|l|}{ Reduced-form Model } \\
\hline Investigators & Date & Investigators & Date \\
\hline Black \& Scholes & 1973 & Geske, Ingersoll, Merton & 1977 \\
\hline Merton & 1974 & Smith \& Warner & 1979 \\
\hline Black \& Cox & 1976 & Cooper \& Mello & 1991 \\
\hline \multirow{3}{*}{\multicolumn{2}{|c|}{ Longstaff \& Schwarts 19? }} & Hull \& White & 1992 \\
\hline & & Abken & 1993 \\
\hline & & Duffie \& Singleton & 1995 \\
\hline
\end{tabular}

As discussed, default risk is expressed through the CDS spread. While pricing of this CDS is a challenging open problem that is a very quantitative and qualitative field involving estimations of default, timing of default and balance sheet value fluctuations (see: $[8,9,12]$ ), the Duffie approach provides a method to evaluate the correct pricing of the CDS spread through the simple relationship.

$$
\text { CDSspread }=\text { RiskFreeRate }- \text { DebtReturn }
$$

Where Risk Free Rate refers to Interest Rate and Debt Return refers to Bond Yield. This paper uses Duffie regression model as the regression benchmark model. This relationship is observed in the market and if it breaks down significantly, traders will buy and sell the instruments to return the relationship close to parity. But observing the CDS spread, debt return and risk free rate in the financial market shows that this relation does not hold exactly. An example of this observation is illustrated in figure 2. The reason of the gap between the Duffie theory and market data is the cost of arbitrage or what is also known as the arbitrage channel [1]. The arbitrage cost comes from the range of market

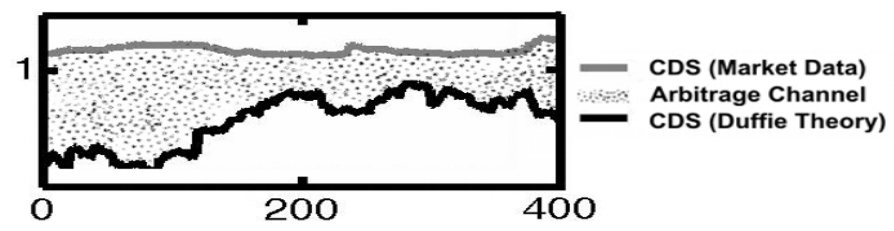

Fig. 2: Duffie Theory Vs. Market Data

mechanics to borrow, sell and buy instruments to profit from the CDS spread, inaccurately estimating the risk of a default event [8]. Therefore, the challenge of the CDS pricing is narrowed to studying of this arbitrage channel and reduce it in order to find a regression model which can match the market data. This paper is focussed on investigating the relationship between CDS spread, debt and equity information using CGP. In analyzing the results of CGP for a common group of financial corporations we expect to discover and propose a new regression model which can estimate the CDS price better than current regression benchmark. 


\section{CGP Model}

CGP was developed from methods developed for the automatic evolution of digital circuits by Miller and Thomson [10] for the purpose of evolving digital circuits. Unlike traditional Genetic Programming (GP), CGP represents a program as a directed (that for feed-forward functions is acyclic) graph. The significance of the difference between CGP and Linear GP has been established in the means of restricting interconnectivity of nodes [16]. In CGP, the genotype is a fixed length representation and consists of a list of integers, which encode the function and connections of each node in the directed graph [15]. The number of nodes in the graph is bounded but it can be varied, as CGP uses a genotype phenotype mapping that allows the existence of unconnected nodes in the genotype which produce inactive sub-genotypes that have no effect on the phenotype. This leads to an effect on search called neutrality, a CGP feature that has been found to be tremendously valuable to the evolutionary process on the problems studied [14]. Each of the nodes is encoded by a number of genes representing a particular function and the inputs that each node has. The nodes take their inputs in a feed forward manner from either the output of a previous node or from one of the initial program inputs (terminals).

CGP has been applied to a growing number of domains and problems such as digital circuit design, digital filter design, image processing, artificial life, bioinspired developmental models, evolutionary art and has been adopted within new evolutionary techniques such as cell-based optimization and social programming. To our knowledge it has not been investigated in the financial field so far while GP is widely used in financial fields such as: Stock markets, Game theory, Betting, Foreign exchange, Arbitrage and Studying markets. See [13] for comprehensive overview of GP and its applications.

\subsection{CGP Model Modification}

For the purpose of this paper objectives the basic CGP model ${ }^{4}$ is modified in order to provide more information from CGP result. Our CGP model has the following features:

1. Training/Test Dataset: Our version of CGP divides the data $X_{a}$ into two datasets where $X_{i}$ randomly chosen data points are considered as the test set and the $X_{a-i}$ remaining data points are considered as the training set. The training set used by CGP as input data, and the best result at the end of the evolutionary run is tested.

2. CGP Output: The original CGP model provide fitness report as the CGP output. For the purpose of analysis our version of CGP reports on training and test sets results which makes the data comparison possible.

3. Chromosome Translator: An important feature of this work is the evolved equation (not just the fitness values). Hence we also have created a solution parser that translates the chromosome into an understandable mathematical equation, which we can then study for insights into the solution.

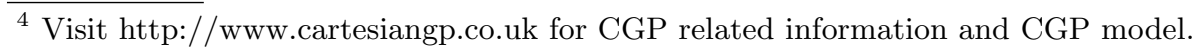




\section{Experimental Datasets, Settings and Objectives}

The Centrica Plc company is chosen for our experiments. The Centrica Plc is a large multinational utility company. It is listed on the London Stock Exchange and also listed on FTSE 100 Index $^{5}$. Data is collected from 5th January 2004 till 25th Jun 2009 (which of course includes the recent highly turbulent nature of the markets). Table 2 illustrates a sample of our database including the company CDS spread, debt and equity information. Two datasets are specified for the system. The first dataset contains three inputs: CDS spread (bp), bond yields (ask and bid price) and Bank of England base rate. The second dataset includes the all available information, eight inputs as shown in table 2 . In the rest of this paper we refer to the first dataset as the CDS-Debt dataset and the second dataset is called CDS-Debt-Equity dataset. Each dataset contains 1400 data points $\left(X_{a}\right)$ where 400 randomly chosen data points $\left(X_{i}\right)$ are considered as the test set and the 1000 remaining data points $\left(X_{a-i}\right)$ are considered as the training set. The test set is the same for all runs.

Table 2: Centrica plc Database (CDS, Debt and Equity Information)

\begin{tabular}{llllllllll}
\hline Date & Spread(bp) & Bid yield & Ask yield & Base rate & Bid PX & Ask PX & E. Volatility & E. Weight & E. PX(High) \\
\hline $05 / 01 / 2004$ & $\mathbf{0 . 2 9 0 0}$ & 5.448 & 5.376 & 3.75 & 102.908 & 103.408 & $\mathbf{7 . 8 8 4 0}$ & $\mathbf{1 8 7 . 7 3 8 1}$ & $\mathbf{1 8 8 . 8 1}$ \\
$\ldots \ldots \ldots$ & $\ldots \ldots$ & $\ldots \ldots$ & $\ldots$ & $\ldots \ldots$ & $\ldots \ldots$ & $\ldots \ldots$ & $\ldots \ldots$ & $\ldots \ldots$ & $\ldots$. \\
$24 / 06 / 2009$ & 0.6667 & 4.172 & 3.990 & 0.50 & 105.188 & 105.768 & 28.996 & 226.5237 & 230.75 \\
$25 / 06 / 2009$ & 0.6652 & 4.157 & 3.972 & 0.50 & 105.232 & 105.822 & 28.840 & 227.5276 & 231.00 \\
\hline
\end{tabular}

Table 3 shows the experimental setup. We run all experiments with the same settings. We vary the mutation rates and the number of nodes (which in CGP affects the overall size of solutions and thus the complexity of equations that can be evolved) in order to monitor CGP behaviour. A simple function set is chosen, containing only fundamental operators as listed in table 3. In addition to our financial inputs, three constant integers (1,2 and 3$)$ have been given as constant inputs to the model as well.

Table 3: CGP Settings

\begin{tabular}{lr|lr}
\hline General Setting & & Function & Symbol \\
\hline Population size: & 5 & Add & + \\
Mutation rate: & $\mathbf{0 . 2 0}, \mathbf{0 . 5 0}, \mathbf{0 . 7 0}$ & Subtract & - \\
No. of generations: & $\mathbf{2 0 0 0 0 0}$ & Multiplication & $*$ \\
No. of runs: & 20 & Division & $/$ \\
No. of rows: & 1 & Power & Pow \\
No. of cols: & 250 or 500 & Square root & Sqrt \\
Levels back: & 250 or 500 & & \\
\hline
\end{tabular}

The fitness is calculated for each datapoint by defining the error rate, calculated as the absolute value of difference between the CGP-Output and the actual data: Error $=\mid C G P_{\text {Output }}-$ Data $\mid$ and converting this result to a number between 0 and 1 where this number demonstrate the portion of the number of actual values that is predicted correctly by CGP: DataPointFitness $=\frac{1.0}{1.0+\text { Error }}$. The fitness of the whole dataset is equal to sum of all data points' fitnesses and the best dataset fitness is equal to number of data points. Thus, a higher fitness means a better result as it shows the smaller error rate. We follow two main objectives in our experiments:

\footnotetext{
${ }^{5}$ FTSE 100 index is a share index of the 100 most highly capitalised UK companies listed on the London Stock Exchange.
} 
1. Monitoring CGP behavior under different settings. Therefore, the first experiments are ran on the CDS-Debt dataset with different combinations of nodes $(500,250)$ and mutation rates $(0.20,0.50,0.70)$ to see how these two factors will affect the results. Following these experiments, the ability of CGP to deal with and distinguish between relevant and irrelevant inputs is examined by using the second dataset (CDS-Debt-Equity dataset) containing more data attributes.

2. Assessing the CGP reliability as regression discovery tool. Of interest, is to observe, whether CGP can come up with a regression model that can price CDS better than the regression benchmark model (Duffie Theory) and to understand something of how that model works.

Each experiment was run for 20 times and 200,000 generations.

\section{Results}

Figure 3 shows the CGP fitness report on CDS-Debt dataset. According to the result, although the number of nodes (graph $\mathrm{C}$ and $\mathrm{D}$ ) and mutation rate (graph $\mathrm{A}$ and $\mathrm{B}$ ) affect the CGP performance in terms of reaching a better fitness in early generations, but it does not have a big impact on the average fitness. This means the better fitness dose not always rely on a larger number of nodes and higher mutation rate (graph B and C). For CDS-Debt dataset, the best fitness was accived by 500 nodes and mutation rate of 0.50 .

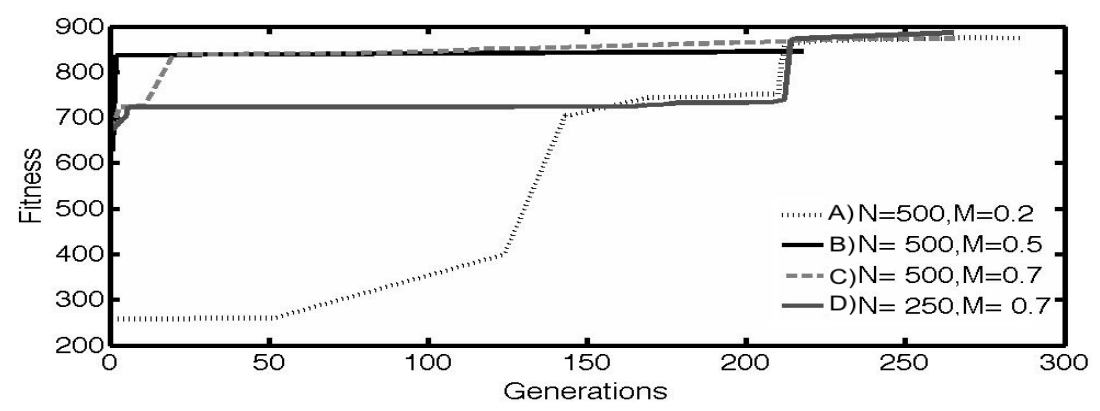

Fig. 3: CGP Behavior Under Different Settings

As we discussed in section 2 one of the important issues of CDS pricing is to reduce the arbitrage channel. Figure 4 and table 4 show the results in terms of accuracy of CDS pricing. In our experiments, CGP discovered a new relationship between bond yield and risk free rate which creates a very accurate prediction of the real CDS price in the market. It also demonstrates the arbitrage gap which exists in Duffie theory is significantly reduced (see figure 4a and 4b). The result shows that the trend of CDS price has been predicted correctly.

In the experiment using the larger dataset (CDS-Bond-Equity dataset), the results show that the extra inputs helped CGP to reduce the arbitrage channel 


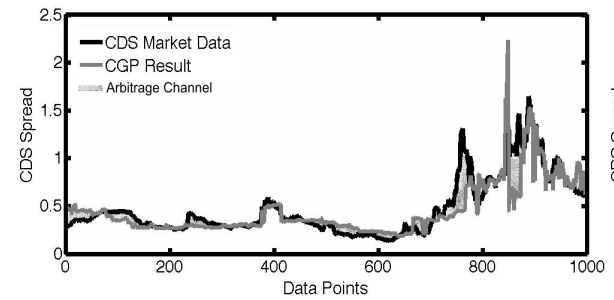

(a) CDS-Bond Training Dataset

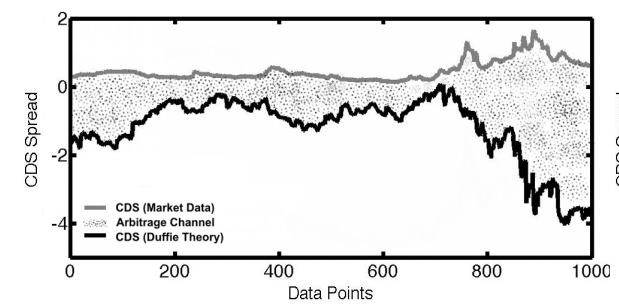

(c) CDS Vs. Duffi Benchmark

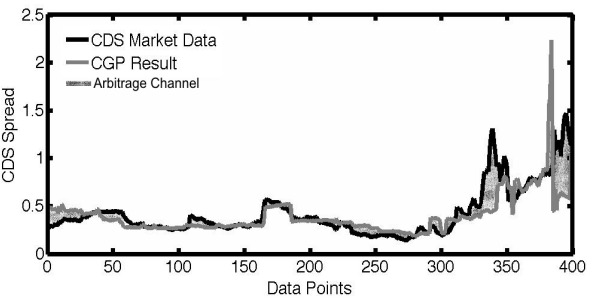

(b) CDS-Bond Test Dataset

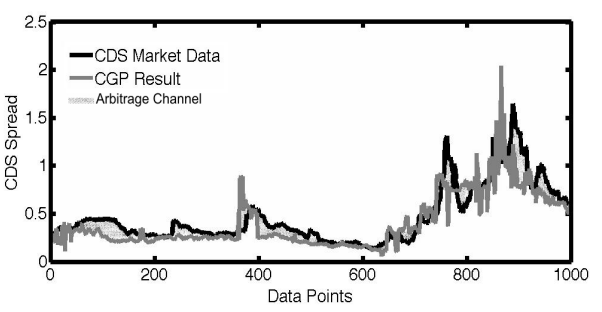

(d) CDS-Bond-Equity Training Dataset

Fig. 4: Experiments Result

Table 4: Error Report

\begin{tabular}{llrr}
\hline Model & Input & Training Set Error & Test Set Error \\
\hline Duffie & CDS-Bond & $167.84928 \%$ & $134.6789 \%$ \\
CGP & CDS-Bond & $8.9769295 \%$ & $9.7533925 \%$ \\
CGP & CDS-Bond-Equity & $10.1807889 \%$ & $10.0130045 \%$ \\
\hline
\end{tabular}

in some parts but it had a negative effect on other parts (see figure 4c and $4 \mathrm{~d}$ ), so the overall error increased, see table 4.

The inability of CGP to perform effective feature selection using this larger number of attributes may be partly because of the complexity of this problem. Some of the additional variables may be useful some of the time and detrimental at other times, meaning that CGP (and indeed any evolutionary algorithm) would find it hard to eliminate them. The complexity of all the relationships, means that an incremental change from a complex solution using more variables into a simpler solution using fewer variables may be impossible without encountering extremely unfit variants, thus making the search unlikely to be successful. Nevertheless, the results are fascinating for they indicate that good accuracy for this problem can be obtained with fewer variables and simpler corresponding equations.

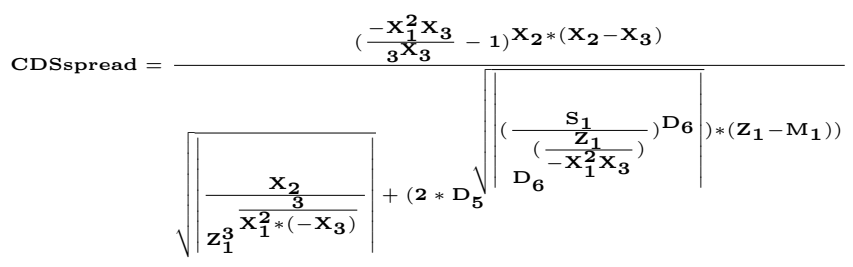


Where

$$
\begin{gathered}
\mathrm{D}_{2}=\left(4-\mathrm{x}_{1}\right) *\left(-\mathrm{X}_{3}^{\mathrm{X}_{2}-1}-\mathrm{M}_{1}\right), \quad \mathrm{D}_{3}=\mathrm{X}_{3}-\mathrm{D}_{2}-\mathrm{x}_{3}, \quad \mathrm{M}_{1}=\mathrm{X}_{2}^{3}+\frac{\mathrm{X}_{2}^{3}}{\mathrm{X}_{3}}, \quad \mathrm{~S}_{1}=\mathrm{X}_{2} *\left(\mathrm{X}_{2}-\mathrm{x}_{3}\right)^{2} \\
\mathrm{D}_{5}=\frac{\mathrm{D}_{3}}{\frac{\mathrm{X}_{1}^{2}}{\mathrm{x}_{1}-\mathrm{X}_{2}-1}} * \mathrm{D}_{4}, \quad \mathrm{D}_{6}=\mathrm{X}_{1}-\frac{3}{-\mathrm{X}_{1}^{2} \mathrm{X}_{3}}, \quad \mathrm{D}_{4}=\frac{\mathrm{D}_{3}}{\mathrm{z}_{1} * 3} * \mathrm{~s}_{1} \quad \mathrm{z}_{1}=\frac{\mathrm{X}_{3}^{3}}{\mathrm{X}_{1}^{2} \mathrm{X}_{3}} \mathrm{X}_{3}
\end{gathered}
$$

Not surprisingly, CGP has evolved completely different equation in each run. Equation (2) shows one of the best evolved solutions. Analysis of all evolved equations reveals that some components are repeated in all solutions. For instance the component $\left(\frac{X_{2}}{X_{3}}\right)$ has been found in 12 best solutions. $X_{2}$ is buying price and $X_{3}$ is selling price of bond yield. Moreover, the component $\left(X_{2}-X_{3}\right)$ which shows the difference amount between sell price and buy price of bond yield is several regions of the equations of 7 best solutions. CGP has discovered these possible relationships between $X_{2}$ and $X_{3}$ (buy and sell prices). To understand the significance of these relationships, we test the affect of these two components by reducing the sell price and buy price difference.

$$
\lim _{\left(X_{2}-X_{3}\right) \rightarrow 0} C D \text { Sspread and } \quad \lim _{\left(\frac{X_{2}}{X_{3}}\right) \rightarrow 1} \text { CDSspread }
$$

The computational results show that the error rate significantly increases by ignoring the difference amount between $X_{2}$ and $X_{3}$ but the theoretical regression benchmark ignored these relationships completely by using the average value of buy and sell price or just one of them.

\section{Conclusions}

CDS pricing is highly significant, not just for finance, but for the world economy. This is one of the first ever investigations into the CDS market using machine learning. In this work we used Cartesian Genetic Programming to derive new relationships between variables in order to produce a dramatically more accurate model for CDS pricing compared to the standard Duffi approach. We demonstrated the effectiveness of this bio-inspired evolutionary method for a complex real-world financial problem. Our data included the highly turbulent behaviour of the markets in the last two years, with no loss of accuracy - a significant improvement over the Duffi method which showed a serious fall in accuracy. We also demonstrated the sensitivity of CGP parameters and showed that CGP was able to provide more consistent results using fewer attributes. Future research will focus on performing more experiments on a comprehensive financial database and more comparison analysis.

Although this may be the first use of CGP in finance, the results are highly significant and revealing. This suggests that other bio-inspired methods designed for noisy, unpredictable and unknown data may also be able to illuminate some of the hitherto murky waters of financial trading. We anticipate with tools such as these, future financial crises may be less likely to occur. 


\section{Acknowledgments}

We would like to acknowledge Julian F. Miller for patiently supporting us over CGP model and also special thanks to Ajay Rathor for providing financial data and kindly sharing his professional financial knowledge and experience.

\section{References}

1. Credit Derivative Credit Derivative Handbook. Merrill Lynch, April 2003.

2. Credit Derivatives Handbook. J.P.Morgan-Corporate Quantitative Research, 2006.

3. O'Kane Et Al. The Lehman Brothers Guid to Exotic Credit Derivatives, Risk. Lehman Brothers, 2003.

4. Fisher Black and John Cox. Valuing corporate securities: Some effects of bond indenture provisions valuing corporate securities: Some effects of bond indenture provisions valuing corporate securities: Some effects of bond indenture provisions. Finance, 1976.

5. Abel Elizalde. Credit default swap valuation: An application to spanish firms. (10.1.1.139.5416), 2005.

6. Bastian Hofberger and Niklas Wagner. Pricing cdx credit default swaps using the hull-white model. 2007.

7. Patrick Houweling and Ton Vorst. Pricing default swaps: Empirical evidence. International Money and Finance, 24:8:1200-1225, 2005.

8. John Hull and Alan White. Valuing credit default swaps: No counterparty default risk. Journal of Derivatives, 8:1:29-40, 2000.

9. Tara Joro and Paul Na. Simulation-based first to default(ftd) credit default swap(cds) pricing approach under jump diffusion. Proceedings of the 36th conference on Winter simulation., pages 1632-1636, 2004.

10. Julian Francis Miller and Peter Tomson. Cartesian genetic programming. Proc of the European Conference on Genetic Programming, pages 121-132, 2000.

11. Arora Navneet, Jeffrey Bohn, and Fanlin zhu. Reduced form vs. structural models of credit risk: A case study of three models. Investment Managment, 3:4:43-67, 2005.

12. Group of Twenty Finance Ministers of Central Bank Governors. Declaration of the summit on financial markets and the world economy.

13. Riccardo Poli, William B. Langdon, Nicholas F. McPhee, and John R. Koza. Genetic programming an introductory tutorial and a survey of genetic programming an introductory tutorial and a survey of techniques and applications. Technical Report CES-475, Available at: http://www.essex.ac.uk/csee/research/publications/ technicalreports/2007/ces475.pdf, October 2007.

14. James Alfred Walker and Julian Francis Miller. Evolution and acquisition of modules in cartesian genetic programming. In Proc of EuroGP, LNCS 3003:187-197, 2004 .

15. James Alfred Walker, Julian Francis Miller, and Rachel Cavill. A multichromosome approach to standard and embedded cartesian genetic programming. Proceedings of the 8th annual conference on Genetic and evolutionary computation, pages 903910, 2006.

16. Garnett wilson and Wolfgang Banzhaf. A comparison of cartesian genetic programming and linear genetic programming. EuroGP, LNCS(4971):182-193, 2008. 